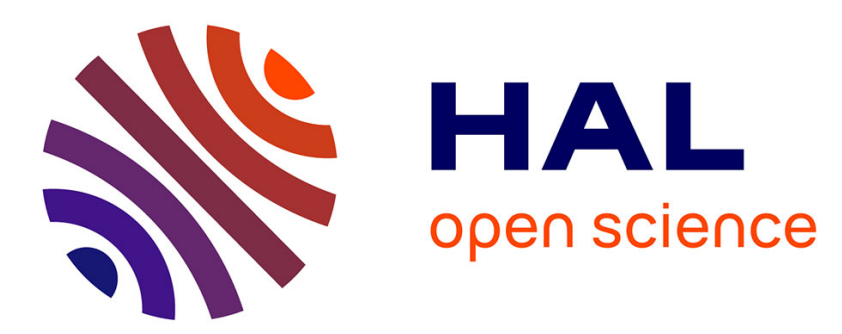

\title{
A New Differential Space-Time Modulation Scheme for MIMO Systems with Four Transmit Antennas
}

\author{
Hui Ji, Gheorghe Zaharia, Jean-François Hélard
}

\section{To cite this version:}

Hui Ji, Gheorghe Zaharia, Jean-François Hélard. A New Differential Space-Time Modulation Scheme for MIMO Systems with Four Transmit Antennas. ICT 2013, May 2013, Casablanca, Morocco. pp.1-5. hal-00826422

\section{HAL Id: hal-00826422 \\ https://hal.science/hal-00826422}

Submitted on 27 May 2013

HAL is a multi-disciplinary open access archive for the deposit and dissemination of scientific research documents, whether they are published or not. The documents may come from teaching and research institutions in France or abroad, or from public or private research centers.
L'archive ouverte pluridisciplinaire HAL, est destinée au dépôt et à la diffusion de documents scientifiques de niveau recherche, publiés ou non, émanant des établissements d'enseignement et de recherche français ou étrangers, des laboratoires publics ou privés. 


\title{
A New Differential Space-Time Modulation Scheme for MIMO Systems with Four Transmit Antennas
}

\author{
Hui Ji, Gheorghe Zaharia, Jean-François Hélard
}

Université Européenne de Bretagne (UEB)

INSA, IETR, UMR 6164, F-35708, Rennes, France

Email:\{hui.ji; gheorghe.zaharia; jean-francois.helard\}@insa-rennes.fr

\begin{abstract}
In this paper, a new differential space-time modulation (DSTM) scheme for $4 \times 4$ multiple input multiple output (MIMO) systems is proposed. This scheme is used for MIMO systems where the channel coefficients are not available at both the transmitter and the receiver. The transmission matrices used in this scheme belong to the Weyl group. Simulation results show that this new scheme with four transmit antennas outperforms the well-known Tarokh's differential space-time block coding (DSTBC) scheme. The spectral efficiency of this scheme can be up to $3 \mathrm{bit} / \mathrm{s} / \mathrm{Hz}$.
\end{abstract}

Keywords-MIMO, DSTM, DSTBC, non-coherent.

\section{INTRODUCTION}

In the late 1990s, due to the large demands of wireless spectral resources, researchers resort to multiple antennas to increase the capacity and robustness of wireless communication systems. Huge amounts of studies have been carried out in this domain. References [1] and [2] analyzed the capacity and the error exponents of such systems in the presence of Gaussian noise and got some fundamental theoretical results. When the throughput is not too high, the estimation of the channel coefficients can be performed easily. Consequently, several schemes with known channel state information (CSI) have been widely analyzed.

In practice, knowledge of the channel is often obtained via training, using signals which are periodically transmitted. However, it is not always feasible or advantageous to use training-based schemes, especially when many antennas are used or when the propagation channel changes rapidly. With multiple input multiple output (MIMO) systems, the number of channels to estimate is equal to the product of the number $M$ of transmit antennas by the number $N$ of receive antennas. Furthermore, the length of the resulting training sequence grows proportionately with the number $M$ of transmit antennas [3], which in turn reduces the overall system throughput. Therefore, solutions that do not require channel information are very interesting in such cases, specifically when the number of transmit and receive antennas are very large.

Marzetta and Hochwald [4] analyzed the capacity of the MIMO systems without CSI. Based on the results, they proposed the unitary space-time modulation scheme (USTM) [5]. In succession, motivated by the single antenna differential phase-shift keying (DPSK) systems, Hochwald and Sweldens proposed the differential unitary space-time modulation scheme (DUSTM) [6]. These two schemes are difficult to design, and furthermore there are not general design criteria for these two schemes. At the same time, Tarokh and Jafarkhani presented a differential space-time block coding (DSTBC) scheme [7] which is based on Alamouti's diversity scheme [8], and soon they expanded this scheme to systems with 4 transmit antennas [9]. This scheme is suitable for MIMO systems with less than 4 transmit antennas and the spectral efficiency is limited to $1 \mathrm{bit} / \mathrm{s} / \mathrm{Hz}$. Hughes also designed a differential space-time modulation scheme in [10]. In [11]-[12], authors invented a new kind of non-coherent space-time modulation scheme-matrix coded modulation (MCM) which is suitable for $2 \times 2$ MIMO systems. In the study of the MCM scheme, we found that the group used in this scheme can be also used for differential MIMO schemes.

In this paper, we propose a new kind of differential space-time modulation scheme which is suitable for MIMO systems with 4 transmit antennas. The simulation results demonstrate the advantages of this new scheme over Tarokh's DSTBC scheme. Furthermore, the modulation of Tarokh's scheme with 4 transmit antennas can only be BPSK [9], which prevents the scheme to expand to higher spectral efficiency. Our scheme with four transmit antennas can be extended to a spectral efficiency up to $3 \mathrm{bit} / \mathrm{s} / \mathrm{Hz}$ (defined in section II).

The following notations are used through the paper: $\operatorname{Tr}\{A\}$ denotes the trace of the matrix $A$ and $A^{H}$ denotes the conjugate transpose of $A .\|A\|$ means the Frobenius norm of $A$, i.e. $\|A\|=\sqrt{\sum_{i, j}\left|a_{i j}\right|^{2}}=\sqrt{\operatorname{Tr}\left\{A^{H} A\right\}}$. $\operatorname{Re}\{z\}$ is the real part of the complex number $z$. The sign $\lfloor K\rfloor$ denotes the nearest integer number less than $K$, and $\otimes$ means the Kronecker product. The zero -mean, unit-variance, circularly symmetric, complex Gaussian distribution is written as $C N(0,1)$.

\section{MIMO SYSTEM MODEL}

The MIMO system model in the matrix form is:

$$
Y_{\tau}=H_{\tau} X_{\tau}+W_{\tau},
$$


where $\tau$ is the time index. $H_{\tau}$ is the channel coefficient matrix at time $\tau$ and its size is $N \times M$, where $M$ is the number of transmit antennas and $N$ is the number of receive antennas. The element $h_{n m}$ is the path gain of the quasi-static channel from the transmit antenna $m$ to the receive antenna $n$, and follows complex Gaussian distribution with zero-mean and unit-variance, i.e. $h_{n m} \sim C N(0,1) . \quad X_{\tau}$ is the $M \times T$ transmission matrix, where $T$ denotes the normalized symbol duration of each matrix, i.e. for each transmit antenna, $T$ symbols are transmitted. $Y_{\tau}$ is the $N \times T$ received matrix. $W_{\tau}$ is the $N \times T$ additive white Gaussian noise matrix, $w_{n t} \sim C N\left(0, \sigma^{2}\right)$ and $\sigma^{2}$ is the power of the noise. We denote the spectral efficiency of the system as expressed in $\mathrm{bit} / \mathrm{s} / \mathrm{Hz}$

In this paper, we assume that the channel coefficients are constant during $L$ symbols. Therefore, we ignore the index $\tau$ of the matrix $H_{\tau}$ and the channel matrix is written as $H$.

Furthermore, we assume a normalized power over $M$ transmit antennas:

$$
\sum_{m=1}^{M}\left|x_{m t}\right|^{2}=1, t=1, \ldots, T .
$$

As proved in [4], for non-coherent MIMO systems, for any block length $T$, any number $N$ of receive antennas and any SNR (signal to noise ratio), the capacities obtained with $M>T$ and $M=T$ are equal. Therefore, we choose $M=T$ in our study.

\section{A. The classic Tarokh's DSTBC scheme}

Using DPSK modulation and Alamouti's transmit diversity scheme [8], Tarokh and Jafarkhani proposed a differential space-time block code [7]. The transmission matrices of Alamouti's scheme have the form:

$$
X_{\tau}=\left[\begin{array}{cc}
s_{2 \tau+1} & -s_{2 \tau+2}^{*} \\
s_{2 \tau+2} & s_{2 \tau+1}^{*}
\end{array}\right],
$$

where the symbols $s_{2 \tau+1}$ and $s_{2 \tau+2}$ are selected from the $2^{b}$-PSK $(b=1,2,3, \ldots)$ modulation constellation:

$$
\left\{\frac{e^{2 k \pi j / 2^{b}}}{\sqrt{2}} \mid k=0,1, \ldots, 2^{b}-1\right\}
$$

where $j=\sqrt{-1}$.

To expand Alamouti's scheme to differential scheme, Tarokh and Jafarkhani resorted to the orthonormal basis representation.

The differential space-time encoding procedure is shown as follows. $2 b$ information bits arrive at the encoder, the first $b$ bits are mapped onto an $2^{b}$-PSK symbol $a_{1}$ and the other $b$ bits are mapped onto $a_{2}$. The symbols $a_{1}$ and $a_{2}$ are then transformed to the differential coefficients $v_{1}$ and $v_{2}$ via a unitary matrix:

$$
\left[v_{1}, v_{2}\right]=\frac{1}{\sqrt{2}}\left[a_{1}, a_{2}\right]\left[\begin{array}{cc}
1 & -1 \\
1 & 1
\end{array}\right]
$$

We can verify that $\left|a_{1}\right|^{2}+\left|a_{2}\right|^{2}=1$ and $\left|v_{1}\right|^{2}+\left|v_{2}\right|^{2}=1$.

The symbols $\left[s_{2 \tau+1}, s_{2 \tau+2}\right]$ transmitted at time $\tau$ are determined by the symbols $\left[s_{2 \tau-1}, s_{2 \tau}\right]$ transmitted at time $\tau-1$ and the mapped differential coefficients $\left[v_{1}, v_{2}\right]$. It can be written as:

$$
\left[s_{2 \tau+1}, s_{2 \tau+2}\right]=v_{1}\left[s_{2 \tau-1}, s_{2 \tau}\right]+v_{2}\left\lfloor-s_{2 \tau}^{*}, s_{2 \tau-1}^{*}\right\rfloor
$$

The transmission matrix at time $\tau$ can thus be written as:

$$
\begin{aligned}
X_{\tau} & =\left[\begin{array}{cc}
s_{2 \tau+1} & -s_{2 \tau+2}^{*} \\
s_{2 \tau+2} & s_{2 \tau+1}^{*}
\end{array}\right] \\
& =\left[\begin{array}{cc}
s_{2 \tau-1} & -s_{2 \tau}^{*} \\
s_{2 \tau} & s_{2 \tau-1}^{*}
\end{array}\right]\left[\begin{array}{cc}
v_{1} & -v_{2}^{*} \\
v_{2} & v_{1}^{*}
\end{array}\right]=X_{\tau-1} V_{\tau}
\end{aligned}
$$

where

$$
V_{\tau}=\left[\begin{array}{cc}
v_{1} & -v_{2}^{*} \\
v_{2} & v_{1}^{*}
\end{array}\right]
$$

is a unitary matrix, i.e. the inverse of the matrix is equal to the conjugate transpose of the matrix. All the possible matrices $V_{\tau}$ form the set $P$. There is a one-to-one map from all the possible $2 b$ information bits to the matrices of the set $P$.

At the receiver side, the received matrix at time $\tau$ is:

$$
\begin{aligned}
Y_{\tau} & =H X_{\tau}+W_{\tau} \\
& =H X_{\tau-1} V_{\tau}+W_{\tau} \\
& =Y_{\tau-1} V_{\tau}+\left(W_{\tau}-W_{\tau-1} V_{\tau}\right)
\end{aligned}
$$

To get the transmitted information bits, we need to estimate the pair of coefficients $\left[b_{1}, b_{2}\right]$, which is equivalent to estimate $V_{\tau}$. Thus, the maximum likelihood demodulation is:

$$
\hat{V}_{\tau}=\arg \min _{V \in P}\left\|Y_{\tau}-Y_{\tau-1} V\right\|=\arg \max _{V \in P} \operatorname{Tr}\left\{\operatorname{Re}\left(Y_{\tau}^{H} Y_{\tau-1} V\right)\right\}
$$

Once the unitary matrix $V_{\tau}$ is got, the inverse mapping is applied and the $2 b$ information bits can be recovered.

However, the references [7] and [9] didn't use this maximum likelihood decoding method. They proposed a linear decoding scheme due to the special structure of Alamouti's scheme. The variable $V$ which is used to decode is linearly scaled by the channel coefficients due to some computations. This method makes the error performance better than the maximum likelihood demodulation based on (9).

\section{NeW DifFERENTIAL SPACE-TIME MODULATION SCHEME}

\section{A. The constellation of the new scheme}

In our scheme, the transmitted matrices are based on the Weyl group $G_{w}$ used in [11]-[12] for matrix coded 
modulation schemes. The Weyl group $G_{w}$ is a set that contains 12 cosets $\left(C_{0}, C_{1}, \ldots, C_{11}\right)$. Each coset contains 16 invertible matrices. The first coset which is a subgroup of $G_{w}$ is defined as:

$$
C_{0}=\left\{\alpha\left[\begin{array}{ll}
1 & 0 \\
0 & 1
\end{array}\right], \alpha\left[\begin{array}{cc}
1 & 0 \\
0 & -1
\end{array}\right], \alpha\left[\begin{array}{cc}
0 & 1 \\
1 & 0
\end{array}\right], \alpha\left[\begin{array}{cc}
0 & 1 \\
-1 & 0
\end{array}\right]\right\}
$$

with $\alpha \in\{1,-1, i,-i\}$. The 12 cosets of $G_{w}$ are derived from $C_{0}$ as follows:

$$
C_{k}=A_{k} \cdot C_{0} \quad \forall k=0,1, \ldots, 11
$$

where the matrices $A_{k}, k=0,1, \ldots, 5$ are respectively:

$$
\begin{aligned}
& A_{0}=\left[\begin{array}{ll}
1 & 0 \\
0 & 1
\end{array}\right], A_{1}=\left[\begin{array}{ll}
1 & 0 \\
0 & i
\end{array}\right], A_{2}=\frac{1}{\sqrt{2}}\left[\begin{array}{cc}
1 & 1 \\
1 & -1
\end{array}\right], \\
& A_{3}=\frac{1}{\sqrt{2}}\left[\begin{array}{cc}
1 & 1 \\
i & -i
\end{array}\right], A_{4}=\frac{1}{\sqrt{2}}\left[\begin{array}{cc}
1 & i \\
1 & -i
\end{array}\right], A_{5}=\frac{1}{\sqrt{2}}\left[\begin{array}{ll}
1 & i \\
i & 1
\end{array}\right],
\end{aligned}
$$

and the matrices $A_{k}, k=6,7, \ldots, 11$ are given by:

$$
A_{k+6}=\eta A_{k}, \text { with } \eta=(1+i) / \sqrt{2} \forall k=0,1, \ldots, 5
$$

Remind that, there are 192 matrices in this group. We number the matrices as $M_{0}, M_{1}, \ldots, M_{191}$. Furthermore, they are all unitary matrices.

\section{B. Differential transmission for MIMO systems with 2 transmit antennas}

Consider a MIMO system with $M=2$ transmit antennas and $N=2$ receive antennas. Each transmit matrix is sent during $T=2$ symbol durations. The number of receive antennas is arbitrary, i.e. we can set $N=1,3, \ldots$. We use the coset $C_{0}$ as the candidate mapping matrices set. There are 16 matrices within the coset $C_{0}$, say $M_{0}, M_{1}, \ldots, M_{15}$, so it is possible to map 4 bits to a given matrix of the coset $C_{0}$. Thus, 4 bits are sent during 2 symbol durations. Hence, the spectral efficiency $R$ is $2 \mathrm{bit} / \mathrm{s} / \mathrm{Hz}$. We can use all the 192 matrices in the group to enlarge the spectral efficiency as shown at the end of subsection III.C.

At time $\tau=0$, we transmit a reference
matrix $X_{0}=M_{0}=\left[\begin{array}{ll}1 & 0 \\ 0 & 1\end{array}\right]$.

Suppose that at time $\tau, X_{\tau}$ is transmitted. At time $\tau+1$, a block of 4 bits arrives. These bits are mapped onto one of the matrices $M_{i_{r+1}}=M_{a}$ of the coset $C_{0}$, and then

$$
X_{\tau+1}=X_{\tau} M_{i_{\tau+1}}
$$

is transmitted. Relation (13) is the fundamental differential transmission relation.
Therefore, the sequence of transmitted matrices is:

$$
\begin{aligned}
& X_{0}=M_{0} \\
& X_{1}=X_{0} M_{i_{1}}=M_{0} M_{i_{1}} \\
& X_{2}=X_{1} M_{i_{2}}=M_{0} M_{i_{1}} M_{i_{2}}
\end{aligned}
$$

$X_{\tau}=X_{\tau-1} M_{i_{\tau}}=M_{0} M_{i_{1}} \cdots M_{i_{\tau}}$

\section{Differential reception for MIMO systems with 2 transmit antennas}

At the receiver side, the antennas received a matrix stream $Y_{0}, \ldots, Y_{\tau}, Y_{\tau+1} \ldots$ We know that

$$
\begin{gathered}
Y_{\tau}=H X_{\tau}+W_{\tau} \\
\text { and } Y_{\tau+1}=H X_{\tau+1}+W_{\tau+1} .
\end{gathered}
$$

Based on the differential transmission relation (13) and (14), we obtain

$$
\begin{aligned}
Y_{\tau+1} & =H X_{\tau+1}+W_{\tau+1} \\
& =Y_{\tau} M_{a}+W_{\tau+1}-W_{\tau} M_{a}, \\
& =Y_{\tau} M_{a}+W_{\tau+1}^{\prime}
\end{aligned}
$$

where $W_{\tau+1}^{\prime}=W_{\tau+1}-W_{\tau} M_{a}$.

Therefore, to estimate the information matrix, the maximum likelihood demodulator is

$$
\begin{aligned}
& \hat{M}_{a}=\arg \min _{0 \leq k \leq 15}\left\|Y_{\tau+1}-Y_{\tau} M_{k}\right\| \\
& =\arg \min _{0 \leq k \leq 15} \operatorname{Tr}\left\{\left(Y_{\tau+1}-Y_{\tau} M_{k}\right)^{H}\left(Y_{\tau+1}-Y_{\tau} M_{k}\right)\right\} \\
& =\arg \max _{0 \leq k \leq 15} \operatorname{Tr}\left\{\operatorname{Re}\left(Y_{\tau+1}^{H} Y_{\tau} M_{k}\right)\right\}
\end{aligned}
$$

As there are $K=192$ matrices in the Weyl group $G_{w}$, for MIMO systems with 2 transmit antennas, the maximum spectral efficiency we can get is

$$
R=\frac{1}{M}\left\lfloor\log _{2} K\right\rfloor=3.5 \mathrm{bit} / \mathrm{s} / \mathrm{Hz}
$$

\section{MIMO systems with 4 transmit antennas}

To design a MIMO system with 4 transmit antennas, the $2 \times 2$ Weyl group is not applicable. We use the Kronecker product to expand the group.

The Kronecker product of two arbitrary matrices $A$ and $B$ is defined as: 


$$
A \otimes B=\left[\begin{array}{ccc}
a_{11} B & \cdots & a_{1 n} B \\
\vdots & \ddots & \vdots \\
a_{m 1} B & \cdots & a_{m n} B
\end{array}\right],
$$

where $A$ is an $m \times n$ matrix, $B$ is a $p \times q$ matrix and the resulting matrix is an $m p \times n q$ matrix. In general, $A \otimes B \neq B \otimes A$ (the Kronecker product is not commutative). The Kronecker product has the properties:

1) $A \otimes B$ is invertible if and only if $A$ and $B$ are invertible:

$$
(A \otimes B)^{-1}=A^{-1} \otimes B^{-1}
$$

2) The operation of transposition is distributive over the Kronecker product:

$$
(A \otimes B)^{T}=A^{T} \otimes B^{T}
$$

With the assumption $M=T$ defined in section II, the transmission matrices for 4 transmit antennas are with size $4 \times 4$.

We have $1922 \times 2$ matrices in the Weyl group, and we want to construct a set of $4 \times 4$ matrices. Due to the properties of the Kronecker product, it is possible to obtain $4 \times 4$ unitary matrices from the matrices of the Weyl group $G_{w}$. There are $192 \times 192=36864$ possible Kronecker products for all the matrices of the Weyl group. Actually we make the Kronecker product between $M_{0}$ and $M_{0}, M_{1}, \ldots, M_{191}$ to get the first 192 possible matrices. Then we make the Kronecker product between $M_{1}$ and $M_{0}, M_{1}, \ldots, M_{191}$ to get the second 192 possible matrices. This procedure is continued to $M_{191}$ to get all the 36864 matrices. After checking these matrices one by one, we verify that there are actually $K=4608$ distinct matrices. They are denoted as $N_{0}, N_{1}, \ldots, N_{4607}$. We call the set of all these $4 \times 4$ matrices $G_{w 4}$. We have verified that the set $G_{w 4}$ is a group.

In our first proposal which is used for $R=1 \mathrm{bit} / \mathrm{s} / \mathrm{Hz}$, we make the Kronecker products between the first matrix $M_{0}=\left[\begin{array}{ll}1 & 0 \\ 0 & 1\end{array}\right]$ of $C_{0}$ and all the matrices in $C_{0}$ to get a set $C_{00}$.

$$
\begin{gathered}
C_{00}=M_{0} \otimes C_{0} \\
C_{00}=\left\{\left[\begin{array}{llll}
1 & 0 & 0 & 0 \\
0 & 1 & 0 & 0 \\
0 & 0 & 1 & 0 \\
0 & 0 & 0 & 1
\end{array}\right], \alpha\left[\begin{array}{cccc}
1 & 0 & 0 & 0 \\
0 & -1 & 0 & 0 \\
0 & 0 & 1 & 0 \\
0 & 0 & 0 & -1
\end{array}\right],\right. \\
\left.\alpha\left[\begin{array}{llll}
0 & 1 & 0 & 0 \\
1 & 0 & 0 & 0 \\
0 & 0 & 0 & 1 \\
0 & 0 & 1 & 0
\end{array}\right], \alpha\left[\begin{array}{cccc}
0 & 1 & 0 & 0 \\
-1 & 0 & 0 & 0 \\
0 & 0 & 0 & 1 \\
0 & 0 & -1 & 0
\end{array}\right]\right\}
\end{gathered}
$$

where $\alpha \in\{1,-1, i,-i\}$.
Four information bits are viewed as a block. The block is mapped onto one of the 16 matrices of $C_{00}$. Once the matrix is obtained, it is used to differentially modulate the previous transmitted matrix to get the current transmission matrix. There are 4 information bits transmitted during 4 symbol durations, thus the spectral efficiency is $R=1 \mathrm{bps} / \mathrm{Hz}$.

In this case, during the transmission procedure, the first transmitted matrix is

$$
X_{0}=M_{00}=M_{0} \otimes M_{0}=\left[\begin{array}{llll}
1 & 0 & 0 & 0 \\
0 & 1 & 0 & 0 \\
0 & 0 & 1 & 0 \\
0 & 0 & 0 & 1
\end{array}\right] .
$$

The constellation of the modulation of this scheme (i.e. the possible values of the matrices' elements) is $\{ \pm 1, \pm i, 0\}$ which corresponds to 4-PSK $\cup 0$, and the spectral efficiency is $1 \mathrm{bps} / \mathrm{Hz}$. For comparison, the $4 \times 4$ DSTBC scheme [12] with BPSK modulation has the same spectral efficiency. However, this new scheme has better BER performance. Furthermore, there are $K=4608$ distinct matrices in the group $G_{w 4}$. The maximum spectral efficiency we can get is $R=\frac{1}{M}\left\lfloor\log _{2} K\right\rfloor=\frac{1}{4}\left\lfloor\log _{2} 4608\right\rfloor=3 \mathrm{bps} / \mathrm{Hz}$. The simulation with different spectral efficiencies are shown in Fig. 2.

For the spectral efficiency $R=2,8$ bits should be transmitted during 4 symbol durations. The information bits are mapped onto one of the $2^{8}=256$ matrices. We select the first 256 matrices from $G_{w 4}$ as the candidate transmission set. For $R=3$, we should transmit 12 bits during 4 symbol durations. Similarly, we select the first 4096 matrices from $G_{w 4}$ as the candidate transmission set.

\section{Simulation Results}

In this section, we present the simulation results of the new differential space-time modulation scheme compared to some existing differential space-time modulation schemes. We assume that the channel is quasi-static, i.e. the channel coefficients do not change during one frame of transmission matrices. In our simulation, the length of the frame is $L=100$ (i.e. the channel coefficients are constant during 100 symbol durations and change to new independent realizations for the next frame).

Fig. 1 shows that, for MIMO systems with 2 transmit antennas, our new scheme performs worse than the scheme Tarokh DSTBC M2N2 [7]. This is because the decoding method of our scheme is a general maximum likelihood decoding without any pre-process, while the variable used to decode in [7] is linearly scaled by the channel coefficients due to some pre-process. However, this new scheme performs better than the corresponding DUSTM scheme [6] when SNR is less than 14dB. This is because the DUSTM is designed for large SNR environments.

For 4 transmit antenna systems, this new system performs better than the original Tarokh DSTBC M4N4 [9]. Compared to [9], $0.57 \mathrm{~dB}$ gain is obtained for a bit error rate (BER) equal to $10^{-4}$. However, for large SNR values, the DUSTM scheme has slightly better performance compared to the new differential scheme. 


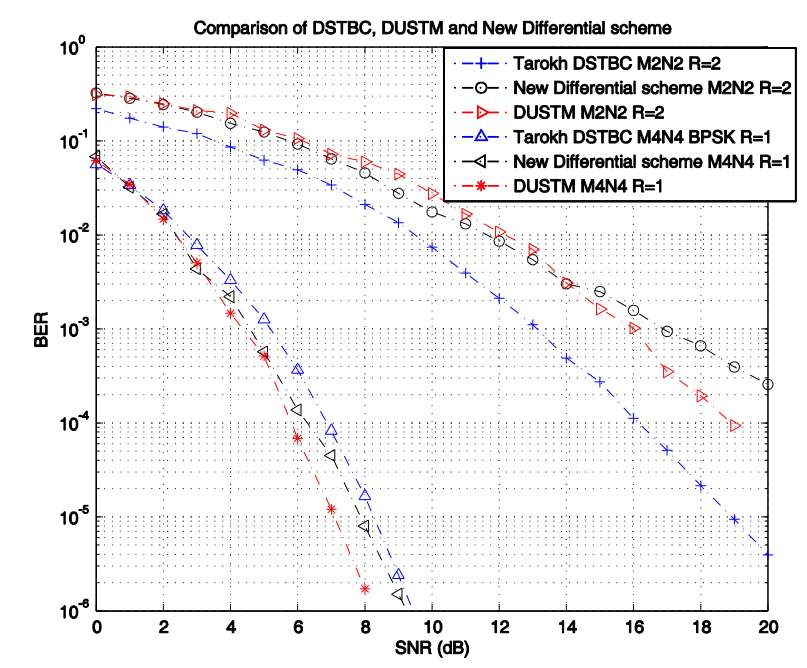

Figure 1. Simulation results of DSTBC [7] [9], DUSTM [6] and our new differential space-time modulation scheme.

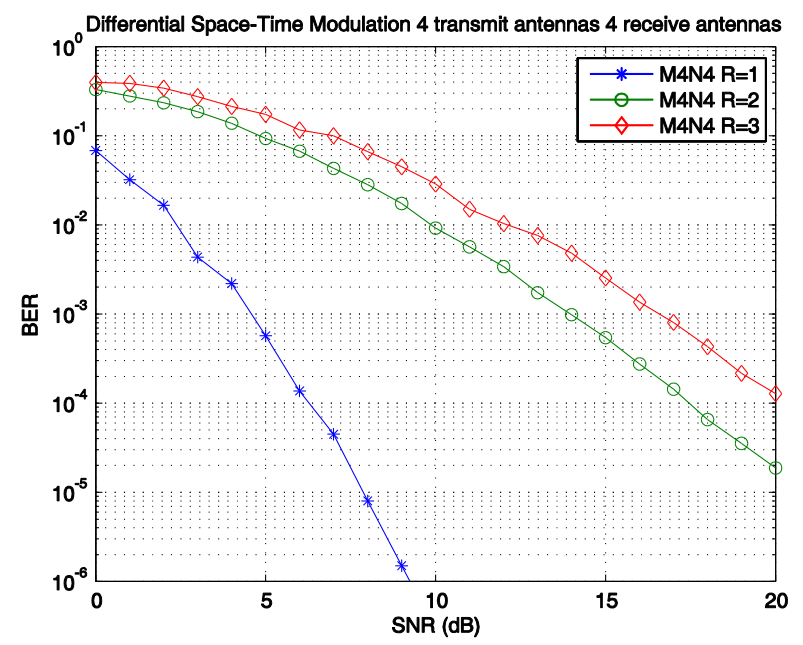

Figure 2. Simulation results of the new differential space-time scheme for 4 transmit antennas and 4 receive antennas with spectral efficiency equal to 1,2 and $3 \mathrm{bit} / \mathrm{s} / \mathrm{Hz}$ respectively.

Fig. 2 shows the BER performance of the new scheme with different spectral efficiencies. It shows that with the spectral efficiency up to $R=3 \mathrm{bit} / \mathrm{s} / \mathrm{Hz}$, the new scheme performs well enough. However, Tarokh's DSTBC scheme can't be expanded to $2^{b}$-PSK $\left(2^{b} \geq 4\right)$ modulations, which prevent it to achieve higher spectral efficiency. The gap from the curve $R=1$ to the curve $R=2$ is quite large. Indeed, for these simulations, the selection of the candidate transmission matrices is arbitrary. This suggests us to study the distance spectrum of the matrices of $G_{w}$ and $G_{w 4}$ in order to select the best set of candidate transmission matrices.

\section{CONCLUSION}

In this paper, a new differential space time modulation scheme based on the Weyl group is proposed for $4 \times 4$ MIMO systems. Simulation results show that this scheme outperforms the well-known Tarokh's differential STBC scheme. The spectral efficiency of this scheme can be increased up to $3 \mathrm{bit} / \mathrm{s} / \mathrm{Hz}$, so higher than $1 \mathrm{bit} / \mathrm{s} / \mathrm{Hz}$ of Tarokh's DSTBC scheme with four transmit antennas.
Furthermore, as in [13]-[15], the performance of this new differential scheme can be improved by selecting the set of transmission matrices having the best distance spectrum. The new scheme can be extended to MIMO systems with more transmit antennas (8 or 16) using the Kronecker product $G_{w} \otimes G_{w 4}, G_{w 4} \otimes G_{w 4}$, etc.

\section{REFERENCES}

[1] E. Telatar, "Capacity of multi-antenna Gaussian channels," AT\&T-Bell Labs Internal Tech. Memo., June 1995.

[2] G. J. Foschini and M. J. Gans, "On limits of wireless communications in a fading environment when using multiple antennas," Wireless Pers. Commun., vol. 6, pp.311 - 335, 1998.

[3] B. Hassibi and B. M. Hochwald, "How much training is needed in multiple-antenna wireless links," IEEE Trans. Inform. Theory, vol. 49, pp. 951-963, Apr. 2003.

[4] T. L. Marzetta and B. M. Hochwald, "Capacity of a mobile multiple-antenna communication link in Rayleigh flat fading," IEEE Trans. Inform. Theory, vol. 45, no.1. pp. 139-157, Jan. 1999.

[5] B. M. Hochwald and T. L. Marzetta, "Unitary space-time modulation for multiple-antenna communications in Rayleigh flat fading," IEEE Trans. Inform. Theory, vol. 46, pp. 543-564, Mar. 2000.

[6] B. Hochwald and W. Sweldens, "Differential unitary space time modulation," IEEE Trans. Commun., vol. 48, pp. 2041-2052, Dec. 2000 .

[7] V. Tarokh and H. Jafarkhani, "A differential detection scheme for transmit diversity," IEEE J. Select. Areas Commun., vol. 18, pp. 1169-1174, July 2000

[8] S. M. Alamouti, "A simple transmitter diversity scheme for wireless communications," IEEE J. Select. Areas Commun., vol. 16, no. 8 pp.1451-1458, Oct. 1998.

[9] H. Jafarkhani and V. Tarokh, "Multiple transmit antenna differential detection from generalized orthogonal designs," IEEE Trans. Inform. Theory, vol. 47, pp. 2626-2631, Sep. 2001.

[10] B. L. Hughes, "Differential Space-Time Modulation," IEEE Trans. Inform. Theory, vol. 16, no. 7, pp. 2567-2578, Nov. 2000.

[11] A. E. Arab, J.C. Carlach and M. Hélard, "A new non-coherent MIMO scheme: Matrix Coded Modulation "MCM"," Communications and Information Technologies (ISCIT), 2011 11th International Symposium on, pp. 120-125, Oct. 2011.

[12] A. E. Arab, J.C. Carlach and M. Hélard, "Joint Space-Time Coded Modulation and Channel Coding for Iterative Non-Coherent MIMO Schemes," Wireless Personal Multimedia Communications (WPMC), 2011 14th International Symposium on, pp.1-5, Oct. 2011.

[13] T.M.H. Ngo, P. Viland, G. Zaharia and J.-F. Hélard, "Balanced QPSK space-time trellis codes," Electron. Lett., vol. 44, no. 16, pp. 983-985, July, 2008.

[14] P. Viland, G. Zaharia and J.-F. Hélard, "Optimal Generation of Space-Time Trellis Codes via Coset Partitioning," IEEE Trans. Vehic. Technol. Vol. 60, no. 3, pp.966-980. March, 2011.

[15] P. Viland, G. Zaharia and J.-F. Hélard, "QPSK super-orthogonal space-time trellis codes with 3 and 4 transmit antennas," Signals, Circuits and Systems (ISSCS), 2011 10th International Symposium on, pp. 1-4, July 2011. 